\title{
SOCIAL SUPPORT PROMOTES MENTAL HEALTH DURING THE COVID-19 OUTBREAK: A CROSS-SECTIONAL STUDY FROM TURKEY
}

\author{
Hatice Kaya ${ }^{1}$, Batuhan Ayık ${ }^{2}$, Rumeysa Tasdelen ${ }^{3}$, Mete Ercis ${ }^{4} \&$ Erhan Ertekin ${ }^{4}$ \\ ${ }^{I}$ Department of Psychiatry, Istanbul Sultanbeyli State Hospital, Sultanbeyli Community Mental Health Center, \\ Istanbul, Sultanbeyli, Turkey \\ ${ }^{2}$ Department of Psychiatry, Istanbul Erenkoy Education and Research Hospital, Istanbul, Erenkoy, Turkey \\ ${ }^{3}$ Department of Psychiatry, Marmara University Istanbul Pendik Education and Research Hospital, \\ Istanbul, Pendik, Turkey \\ ${ }^{4}$ Department of Psychiatry, Istanbul University, Istanbul Faculty of Medicine, Istanbul, Fatih, Turkey
}

received: 13.11.2020;

revised: 25.2.2021;

accepted: 8.3.2021

\section{SUMMARY}

Background: Coronavirus-19 (COVID-19) has several negative effects on mental health, given its rapid transmission, hygiene and isolation measures and associated social and financial difficulties. In this study, we aimed to investigate the mental health burden of the Turkish population and vulnerable groups during the COVID-19 outbreak, especially exploring the effects of social support.

Subjects and methods: We assessed depression, anxiety, stress symptoms and perceived social support among 894 people all over Turkey, using the Depression Anxiety Stress Scales (DASS-21) and Multidimensional Scale of Perceived Social Support (MSPSS) two months after the declaration of the first COVID-19 case in Turkey.

Results: According to DASS-21 subscale scores, the findings showed that the prevalence of moderate to severe depression, anxiety and stress-related symptoms was $24.8 \%, 21.9 \%$ and $12.6 \%$, respectively. Female gender, being single, having a lifetime psychiatric disorder, lower education level and financial concerns were significantly associated with higher DASS-21 scores. Also, there was a negative correlation between all subgroups of perceived social support, especially from the family, and the total DASS-21 score.

Conclusions: Vulnerable groups should be identified and protected to reduce adverse psychiatric outcomes of COVID-19. Besides, further strategies should be provided to maintain protective factors, such as social support, under stressful conditions.

Key words: depression - anxiety - stress - social support - coronavirus

\section{INTRODUCTION}

At the end of December 2019, early cases of acute severe pneumonia and lung failure were reported in Wuhan, China, and the disease was named later as Coronavirus disease 2019 (COVID-19) by the World Health Organization (WHO 2020). The disease which was spread worldwide within a few months has been a growing global public health concern because of its short incubation period, rapid transmission, unclear nature of the symptoms, lack of effective treatment and vaccine, extensive precautionary hygiene and isolation measures and, related financial burdens (De Sousa et al. 2020, Jakovljevic et al. 2020, Yoo 2020). The first confirmed case of COVID-19 disease was reported on 10 March 2020 in Turkey. On 21 March 2020, curfew was declared for elderly over age 65 and for people with chronic illnesses. Later, it was expanded to include people under the age of 20, on 3 April 2020. Additionally, the first curfew for the general population in 31 big cities was announced on 10-12 April 2020 by the Turkish government and it was repeated at all weekends and holidays until June 2020. As of 10 May 2020, 138.657 confirmed cases and 3786 deaths were declared by the Ministry of Health in Turkey (Republic of Turkey Ministry of Health 2020).
Studies from previous outbreaks, such as the Severe Acute Respiratory Syndrome (SARS), Mediterranean Acute Respiratory Syndrome (MERS) and Ebolavirus disease, demonstrated that the society suffered not only from physical symptoms but also a wide range of mental health issues (Chong et al. 2004, Gershon et al. 2016, Yeung \& Fung 2007). A recent review about psychological responses towards outbreaks compiled the salient themes as anxiety, depression, anger, guilt, grief and loss, post-traumatic stress, and stigmatization (Chew et al. 2020). Similarly, a recent web-based search investigating the psychological impact of the COVID-19 outbreak in China revealed that the overall prevalence of general anxiety disorder and depressive symptoms were $35.1 \%$ and $20.1 \%$, respectively (Huang \& Zhao 2020a). Another study from China, which was conducted with 1210 participants, demonstrated that the prevalence of symptoms of moderate to severe depression, anxiety, and distress were found to be $16.5 \%, 28.8 \%$, and $8.1 \%$, respectively (Wang et al. 2020).

During the COVID-19 outbreak, factors such as the rapid spread of the disease, strict social isolation measures, financial concerns, lack of social support, increased use of social media and the impact of the "infodemic" (i.e. excessive information about the 
outbreak which makes it difficult to identify solutions) should also be considered when assessing mental health problems (Cao et al. 2020, De Sousa et al. 2020, Guan et al. 2020, Hu et al. 2020, Xiang et al. 2020). Furthermore, there are some vulnerable populations more likely to be affected by the negative outcomes of the outbreak. In this context, recent studies have shown that being female, young, or a healthcare worker, having pre-existing mental disorders, lower average household income, education level and social support were associated with higher rates of anxiety and depression (Wang et al. 2020).

Several coping mechanisms were defined which help the individuals to cope with these psychological effects of the outbreaks, seeking social support is one of them (Chew et al. 2020). Likewise, recent studies demonstrated that people with lower social support is particularly vulnerable to the negative outcomes of the COVID-19 outbreak (Cao et al. 2020, Wang et al. 2020, Xiao et al. 2020, Zhang et al. 2020).

Although the recession period of the outbreak has started in many countries, it is crucial to understand the mental health burden of the disease, considering the possibility of the second wave of the COVID-19 in the coming months or other potential outbreaks. Therefore, this study aimed to investigate the psychological outcomes of the COVID-19 outbreak in the general Turkish population and associated factors, including social support. We hope our study will contribute to the efforts of identifying psychological challenges, related factors, and vulnerable populations, during the COVID-19 outbreak.

\section{SUBJECTS AND METHODS}

\section{Research design and participants}

A cross-sectional survey was designed to assess the psychological effects of the outbreak of COVID-19 on the general population using an online questionnaire. We collected the data between $10^{\text {th }}$ and $21^{\text {st }}$ of May 2020, starting two months after the declaration of the first COVID-19 case in Turkey. COVID-19 timeline in Turkey, including the period of the collecting data is shown in Figure 1.

Turkish citizens aged over 18 years old were invited to participate in the online survey using a snowball sampling strategy. Eight hundred and ninetyfour participants from all over the country were involved in the current study. All respondents provided informed consent at the beginning of the survey by confirming their willingness to participate in this study. Ethical approval for this study was obtained by the ethical committee at Istanbul Marmara University with the number 2020/500.

\section{Measurements}

The study questionnaire included six main components as follows: socio-demographic characteristics (gender, age, marital status, education, working status, monthly income and living in cities with curfew or in other cities), previous psychiatric or physical diseases, changes in nicotine, alcohol and social media use, concerns about COVID-19 outbreak, the Depression, Anxiety, Stress Scale-21 (DASS-21) and the Multi-Dimensional Scale of Perceived Social Support (MSPSS).

Psychological symptoms, such as depression, anxiety and stress, were assessed using DASS-21, which is a 4-point Likert-type screening instrument and demonstrated to be reliable and valid in the Turkish population (Lovibond \& Lovibond 1995, Y1lmaz et al. 2017). The self-reported 21-item scale provides independent measures of depression, stress, and anxiety with recommended severity thresholds for depression, stress, and anxiety subscales. The scores for each of the three components were calculated by summing up the scores for the relevant items and multiplying by two to calculate the final score. Cut-off scores are 9, 7 and 14 for depression, anxiety, and stress, respectively. The severity of depression, anxiety, or stress is also assessed by subscale cut-off scores (Antony et al. 1998).

The Turkish version of the Multi-Dimensional Scale of Perceived Social Support (MSPSS) was used to measure the subjective assessment of perceived social support. The MSPSS developed in 1988 by Zimet et al., is a 12-item scale that consists of three subscales as follows: Family, friends, and significant others. Each subscale comprises four items, and a seven-point Likert scale is used to score the items ( $1=$ very strongly disagree to $7=$ very strongly agree) (Zimet et al. 1988). Higher scores indicate a greater perception of social support. The total score of the scale varies between 12 and 84 . The Turkish adaptation and determination of the validity and reliability of the scale were performed by Eker and Arkar in 1995 (Eker \& Arkar 1995).

\section{Statistical analyses}

Statistical Package for Social Sciences, version 20 (SPSS Inc., Chicago, IL) was used to analyze the data. Results were given as mean \pm standard error for continuous variables and as count and percentages (\%) for categorical variables. A P-value of less than 0.05 was considered statistically significant, and all analyses were two-tailed. Univariate analysis to compare continuous variables like DASS-21 or MSPSS scores was conducted by using Student T-test or One-Way ANOVA. According to the homogeneity of the variance, Tukey or Tamhane's T2 test was used in the post-hoc pairwise comparison of multiple groups. Pearson's correlation test was used for the continuous variables, and Spearman's rank correlation test was used for ordinal variables for correlation analysis. Multiple linear regression analysis was conducted to investigate the unique effects of different variables on the total DASS-21 score. 


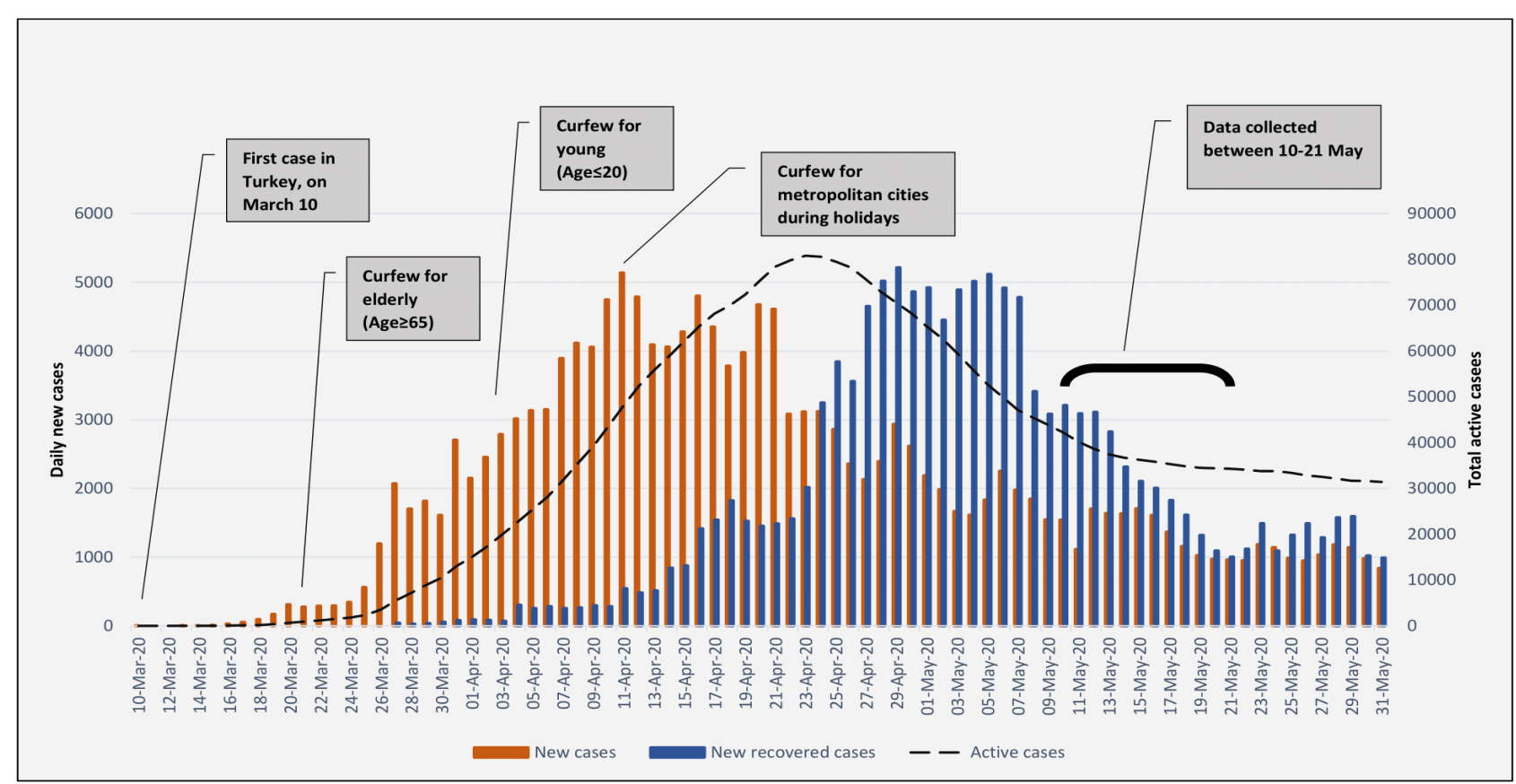

Figure 1. Coronavirus Outbreak Timeline in Turkey

\section{RESULTS}

\section{Sociodemographic variables}

All data were collected between May 10 and May 21, 2020. Our sample consisted of 894 subjects after we excluded three subjects with missing data. $52.6 \%$ $(n=470)$ of the participants were female and mean age was $39.09(\mathrm{SD}=12.9) .64 .8 \%(\mathrm{n}=579)$ of the subjects were married and $58.8 \%(\mathrm{n}=526)$ had at least one child. Most the participants $60.5 \%(\mathrm{n}=541)$ had undergraduate degree, $18.2 \%(n=163)$ had graduate and $16.1 \%(n=144)$ had high school degree. $87.9 \%(n=786)$ of the subjects stated that they were living in the cities with curfew declared due to pandemic. Most of the participants (63.3\%, $\mathrm{n}=566)$ were living with their spouse and/or children, 26.2\% $(\mathrm{n}=234)$ were living with their parents and $8.4 \%(\mathrm{n}=75)$ were living alone. $62.0 \%(\mathrm{n}=554)$ were smoking and $30.5 \%(\mathrm{n}=273)$ were using alcohol. While only $9.1 \%(n=81)$ of the participants stated that they had to leave their jobs or had to take unpaid leave during this period, $54.7 \%(\mathrm{n}=489)$ had switched to parttime or online working, and $36.2 \% \quad(n=324)$ were working in the same condition. While $18.6 \%(n=166)$ of the participants indicated that they do not have any income, $12.2 \%(n=109)$ of them stated that their income was around the minimum wage in Turkey. The rates of not feeling safe during work, not feeling safe about their income, and having difficulty in obtaining adequate masks were $37.8 \%(n=338), 46.6 \%(n=417)$ and $37.5 \%$ $(n=335)$, respectively. $23.8 \%(n=213)$ of the participants in the study had a history of a psychiatric disorder in the past and $21.4 \%(n=191)$ had a medical risk factor such as a chronic disease or pregnancy. $12.3 \%(n=110)$ of the participants stated that they had at least one COVID-19 symptom, while only $3.8 \%(n=34)$ stated that they had
COVID-19 testing, among them $11.8 \% \quad(n=4)$ were positive. Those who were subject to quarantine or been in isolation were $5.7 \%(n=51) .4 .4 \% \quad(n=39)$ of the participants had a family member with positive COVID19 testing or been in quarantine.

\section{Results of the DASS-21 scores, MSPSS scores and comparison with sociodemographic variables}

Mean depression, anxiety, stress levels and total DASS-21 scores and perceived social support scores are shown in Table 1. Predetermined cut-off values of the DASS-21 scale were used to assess whether there was at least moderate depression, anxiety, or stress. A quarter of our sample had at least moderate depression and onefifth had at least moderate anxiety.

Table 1. Depression, anxiety and stress levels measured with DASS-21 and Perceived Social Support $(N=894)$

\begin{tabular}{lcc}
\hline & N/Mean & $\% / \mathrm{SD}$ \\
\hline Moderate to very-severe depression* & 222 & $24.8 \%$ \\
Moderate to extremely-severe anxiety* & 196 & $21.9 \%$ \\
Moderate to extremely-severe stress* & 113 & $12.6 \%$ \\
Depression subscale score & 4.31 & 4.15 \\
Anxiety subscale score & 2.66 & 3.19 \\
Stress subscale score & 4.95 & 4.02 \\
Total DASS-21 score & 11.93 & 10.13 \\
Social support (family) & 23.32 & 5.49 \\
Social support (friends) & 21.89 & 6.27 \\
Social support (significant others) & 18.77 & 8.36 \\
Social support Total & 63.97 & 15.92 \\
\hline
\end{tabular}

DASS-21: Depression, Anxiety and Stress Scale - 21 Items *Cut-off according to Manual for the Depression Anxiety Stress Scales (Lovibond \& Lovibond 1995) 
Comparison of the mean depression, anxiety, stress levels and total DASS-21 score according to sociodemographic variables and other various factors associated with the COVID-19 outbreak are shown in Table 2. Female gender, marital status of being single, having a lifetime psychiatric disorder, history of chronic illness or having other risk factors, lower education level, lower monthly income, job loss, or getting unpaid leave were significantly associated with higher DASS-21 scores. Also, there was a negative correlation between age and DASS-21 scores, although the relationship was low. City of residency, being tested for COVID-19, history of quarantine or isolation, having a family member who tested positive for COVID-19 or been in quarantine had no significant effect on total DASS-21 or its subscale scores.

Having children (10.567 \pm 9.549 vs. $13.867 \pm 10.624$, $\mathrm{t}=4.763, \mathrm{p}<0.001)$ and living with someone else $(11.631 \pm 9.903$ vs. $15.133 \pm 11.955, \mathrm{t}=2.461, \mathrm{p}<0.016)$ were associated with lower total DASS-21 scores.

Further univariate analysis was conducted for total DASS-21 score and change in smoking habits (only in smokers), alcohol consumption habits (only in alcohol users) and social media use patterns (Table 3). Increase in social media usage was correlated with total DASS21 scores $(\mathrm{r}=0.205, \mathrm{p}<0.001)$.

There was a negative correlation between total social support score and total DASS-21 score ( $\mathrm{r}=-0.224$, $\mathrm{p}<0.001)$. This was still significant after controlling for age $(r=-0.227, p<0.001)$. All subgroups of social support were correlated with total DASS-21 score. Social support from the family had the greatest effect size among subscales (Family: $r=-0.277, p<0.001$ vs. Friends: $r=-0.168$, $\mathrm{p}<0.001$ and Significant other: $r=-0.118, \mathrm{p}<0.001)$.

We later conducted multiple linear regression analysis on DASS-21 total score on independent sociodemographic variables and perceived social support. Our regression model included variables that were statistically significant in a univariate analysis like age, gender, marital status, having children, education level, household status (living alone or with someone), monthly income, history of psychiatric disorder and perceived social support. However, we excluded having a child and household status as they were highly correlated with marital status. Our final model accounted for $17.9 \%$ of the variance in total DASS-21 score $(F(7.886)=27.65, \quad \mathrm{p}<0.001$, $\left.R^{2}=0.179\right)$ and is shown in Table 4. Female gender, younger age, being single, lower education level, history of

Table 3. Change in smoking, alcohol consumption habits and social media use patterns

\begin{tabular}{|c|c|c|c|c|c|}
\hline & & $\mathrm{N}$ & Mean & SD & $\mathrm{p}$ \\
\hline \multirow[t]{4}{*}{ Smoking } & No change & 175 & 12.2457 & 9.50452 & \multirow{4}{*}{$\mathrm{p}=0.005^{*}$} \\
\hline & Less than usual & 93 & 13.8817 & 11.25674 & \\
\hline & More than usual & 45 & 18.3556 & 10.64330 & \\
\hline & Quit smoking & 27 & 12.1481 & 10.90767 & \\
\hline \multirow[t]{4}{*}{ Alcohol consumption } & No change & 142 & 12.8169 & 8.84189 & \multirow[t]{4}{*}{$\mathrm{p}=0.060$} \\
\hline & Less than usual & 80 & 14.7250 & 10.91854 & \\
\hline & More than usual & 30 & 17.2667 & 12.67072 & \\
\hline & Stopped & 21 & 17.1429 & 10.37924 & \\
\hline \multirow[t]{4}{*}{ Social media use } & Less than usual & 35 & 8.9143 & 8.30794 & \multirow[t]{4}{*}{$\mathrm{p}<0.001 * *$} \\
\hline & As usual & 301 & 9.6146 & 9.41900 & \\
\hline & More than usual & 415 & 12.1181 & 9.80969 & \\
\hline & Much more than usual & 143 & 16.9650 & 11.03223 & \\
\hline
\end{tabular}

* Post-hoc pairwise comparison showed that individuals who smoked more than usual had higher total DASS-21 scores than individual with no change in smoking habits; $\quad * *$ Post-hoc pairwise comparison showed that individuals who used social media more had higher total DASS-21 scores than individual with less or as usual use. Individual who used social media much more had higher total DASS-21 scores than all other groups.

Table 4. Multiple regression analysis of total DASS-21 score and sociodemographic variables

\begin{tabular}{|c|c|c|c|c|c|c|c|}
\hline & \multirow{2}{*}{$\mathrm{B}$} & \multirow{2}{*}{$\mathrm{SE}$} & \multirow{2}{*}{$\mathrm{B}$} & \multirow{2}{*}{$\mathrm{T}$} & \multicolumn{2}{|c|}{$95 \% \mathrm{CI}$} & \multirow{2}{*}{$\mathrm{p}$} \\
\hline & & & & & LL & UL & \\
\hline (Constant) & 23.812 & 1.765 & & 13.491 & 20.348 & 27.276 & 0.000 \\
\hline Age (years) & -0.066 & 0.030 & -0.081 & -2.206 & -0.124 & -0.007 & 0.028 \\
\hline Gender & -2.352 & 0.651 & -0.116 & -3.613 & -1.074 & -3.629 & 0.000 \\
\hline Marital Status & -2.714 & 0.745 & -0.128 & -3.644 & -4.177 & -1.252 & 0.000 \\
\hline Education level $^{3}$ & -2.271 & 0.832 & -0.092 & -2.731 & -3.903 & -0.639 & 0.006 \\
\hline Monthly income ${ }^{4}$ & -0.039 & 0.343 & -0.004 & -0.114 & -0.712 & 0.634 & 0.910 \\
\hline History of psychiatric disorder ${ }^{5}$ & 5.278 & 0.732 & 0.222 & 7.208 & 3.841 & 6.715 & 0.000 \\
\hline Perceived social support $^{6}$ & -0.127 & 0.020 & -0.200 & -6.429 & -0.166 & -0.089 & 0.000 \\
\hline
\end{tabular}

$\mathrm{B}=$ Unstandardized Beta; SE: Standard Error; $\beta$ : Standardized Beta Coefficient; CI: Confidence Interval

1: $0=$ Female, $1=$ Male; ${ }^{2}: 0=$ Single, $1=$ Married; $3: 0=$ Highschool degree or less, $1=$ Undergraduate degree or more;

4: $0=$ No income, $1=$ Minimum wage, $2=$ Twice minimum wage, $3=$ At least thrice minimum wage; $5: 0=$ No, $1=$ Yes;

6 : Measured by the Multidimensional Scale of Perceived Social Support 
lifetime psychiatric disorder and lower social support were independently associated with a more negative psychiatric outcome. According to the results of multiple regression analysis, we found that monthly income was not associated with total DASS-21scores.

\section{Stress factors associated with DASS-21 scores}

In this study, the participants were asked how much the following stress factors affected them on a five-point Likert scale: News about the pandemic, social isolation, other people's disregard for social isolation, curfew, travel restrictions between cities, and financial concerns. Total DASS-21 score was correlated with every stress factor, but the relationships were low, the highest relationship was between total DASS-21 score and 'news about outbreak' ( $r=0.207, \mathrm{p}<0.001)$. Most of the stress factors were correlated with each other, then again, the effect size was small, and the highest effect size was between 'social isolation' and 'other people's disregard for social isolation' $(r=0.254, \mathrm{p}<0.001)$.

\section{DISCUSSION}

As a result of the rapid spread of severe acute respiratory syndrome-associated coronavirus 2 (SARS-CoV2) all over the world, many countries were caught off guard and plenty of measures were taken to prevent the spread of the virus. Globally implementing preventive and controlling measures led to significant changes and restrictions in people's daily lives. There are many studies reporting increased psychological burden of people during the COVID-19 outbreak (Ahmed et al. 2020, Lei et al. 2020, Rajkumar 2020, Reznik et al. 2020). Similarly, in our study, we investigated depression, anxiety, stress levels and related factors in Turkish society during the outbreak and found that $24.8 \%$, $21.9 \%$ and $12.6 \%$ of the participants reported moderate to severe depression, anxiety, and stress symptoms, respectively.

Vulnerable groups were identified concerning the increased psychological burden during the COVID-19 outbreak. Many studies indicated that female gender, being single and younger age were associated with higher rates of anxiety and depression (Huang \& Zhao 2020b, Lei et al. 2020, Liu et al. 2020, Reznik et al. 2020). COVID-19 symptoms are more severe in the elderly population (Zhou et al. 2020), on the other hand, recent studies reported that younger people are more likely to have anxiety and depressive symptoms during the COVID-19 outbreak than older participants due to increased social media exposure and times spent thinking about COVID-19, which is consistent with the findings of our study. Moreover, there was a significant association between financial concerns and younger age. It was shown that individuals who lose their income or job are under the risk of mental health problems, for instance, in a study published in 2020, increased unemployment rates due to the economic crisis were associated with higher suicide mortality rates (Kawohl \& Nordt 2020) . Another vulnerable group is a population with previous psychiatric issues. It was shown in several studies that people with pre-existing psychiatric disorders represent a risky population in the outbreak (Hao et al. 2020, Peteet 2020, Yao et al. 2020). Both disruption of routine psychiatric examinations and the increased stress burden may lead to a worsening of symptoms and exacerbating pre-existing psychiatric disorders as expected (Yao et al. 2020). It has been revealed that people with chronic diseases are more affected by COVID-19 disease (Zhou et al. 2020). A recent study conducted in Turkey has shown that people with accompanying chronic diseases also had psychiatric problems, such as health anxiety (Özdin \& Bayrak Özdin 2020). Similar to these results from recent studies stated above, we found that female gender, younger age, being single, history of psychiatric illness, accompanying chronic diseases, lower education level and lower income were associated with negative psychiatric outcomes.

Recent studies investigating the psychological effects of quarantine and isolation revealed conflicting results. A study conducted in China (Lei et al. 2020) found that quarantine and isolation had significantly increased levels of anxiety and depression of the participants. In another study from China, it was stated that people under quarantine or isolation might experience stressful experiences, such as loss of face-toface communication, not being able to perform their daily social activities, resulting in feelings of loneliness and anger (Ahmed et al. 2020). However, another recent study showed that depression and anxiety symptoms of the participants under quarantine for at least 10 days did not differ from the participants who were not under quarantine (Zhu et al. 2020). In our study, there was no significant difference in DASS-21 total or subgroup scores between groups in terms of whether or not being tested for COVID-19, a history of quarantine or isolation, having a family member who had COVID-19 or been in quarantine. This might be related to the lower number of participants being tested or quarantined. Furthermore, as of May 10, when our study started to be carried out, some steps have been taken to move on to the re-opening phase. From this perspective, the relatively mild course of the outbreak in the country during those days may lead to positive effects on psychiatric outcomes and reduced the negative mental effects of quarantine and isolation. In addition, because of assessing psychiatric symptoms not on time but after the quarantine or COVID-19 testing may also explain the insignificance.

We found that participants with increased social media usage had significantly higher total DASS-21 scale scores. Similarly, there are studies in the literature reporting that increased social media use in the COVID19 outbreak is associated with negative psychiatric outcomes. In a study conducted in Taiwan, it was stated that the main source of information in the outbreak was 
the internet, and this was associated with negative psychological consequences (Ko et al. 2020). In another study, it was reported that excessive information flow especially through social media- over the internet triggers stress and anxiety reactions, and as a result, behaviors leading to suicide attempts could be observed (Ahmed et al. 2020). Besides social media use, other habitual behaviors changed during the outbreak under stressful conditions. Post-hoc pairwise comparison showed that individuals who smoked more than usual had higher total DASS scores than individuals with no change in smoking habits. This result suggests that participants with increased psychological burden smoke more than usual to relieve themselves.

Some recent studies have shown that decreased social support is associated with negative psychological outcomes during the COVID-19 outbreak (Cao et al. 2020, Torales et al. 2020). However, the precautionary regulations on lifestyle (e.g., postponement and cancellation of religious, sports, cultural and entertainment events, lockdown curfew, travel restrictions, self-isolation, social distancing, and quarantine) have hardened to reach the social support desired. In our study, there was a negative correlation between total perceived social support and total DASS-21score. This was still valid when it was controlled for age. All subgroups of social support were correlated with the total DASS-21 score. Social support from the family had the greatest effect size among subscales. Besides this, most of the participants stated that they experienced the fear that their family or loved ones would become sick during the outbreak $(n=647,72 \%)$.

In the face of a pandemic, the medical literature is in need of international and interdisciplinary research. Multisite and multinational research projects are needed to compare data from different sites and countries, so that more can be learned on both local and universal characteristics of COVID-19 (Yelin et al. 2020). To our knowledge, this is the first study in Turkey investigating the association between psychiatric symptoms and social support during the COVID-19 outbreak that can contribute to these international research needs by providing data from a different cultural and religious background.

In addition, the association between psychiatric symptoms and social support might have clinical implications for the clinicians when dealing with their patients who have mental health issues related with COVID-19. For instance, instead of solely focusing on symptoms of depression or anxiety, the clinicians should add an assessment of the social support for their patients into their routine interviews and search for new ways that will improve their patients' social support in order to provide better mental health care. In addition, our study helped to better establish several risk factors associated with more depression, anxiety and stress symptoms during the outbreak such as female gender, being single, having low income etc. The clinicians need to keep in mind the more vulnerable populations for developing psychiatric problems during the outbreak in order not to omit serious psychiatric sequelae of COVID-19.

Family relations are strong in the cultural life of Turkish society. Many people live in family apartments and communicate face to face in their daily lives. It was thought that deprivation of social support due to the precautionary measures and inability to spend face to face time with families could have negative effects on people's psychological well-being. Turkey has a collectivistic culture in which kinship connectedness and social relationships are quite fundamental to social behaviors and psychological well-being in general. Our study has been carried out on a national level. However, it might have implications for similarly collectivistic cultures of the Middle East or Asia. Although social support plays a key role for well-being in any culture, samples from more individualistic Western cultures might reveal different findings than ours. International comparisons - or better yet, multinational studies- might identify the differential psychological effects of social support on a global level during the COVID-19 outbreak.

Our study has several limitations. Firstly, as minimizing face to face interaction is recommended during COVID-19, an online study was designed. Therefore, psychiatric symptoms could not be verified by mental health professionals. Secondly, the study population may not represent all the Turkish population because the snowballing sampling strategy does not give the opportunity to select the participants randomly, leading to selection bias. In addition, as this is a cross-sectional study, the causality is not clear. A position paper stated that research is urgently needed to help understand the mental health consequences of the COVID-19 outbreak (Holmes et al. 2020). We designed a cross-sectional study as we sought to produce results rapidly since there is an urgent need for the proliferation of scientific literature on COVID-19. However, we acknowledge the fact that longitudinal follow up studies are needed to better establish the effect of COVID-19 on mental health.

As there is a possibility of having a second wave of COVID-19 outbreak or experiencing other outbreaks, it is essential to assess psychiatric difficulties that people are going through and develop various preventive strategies for vulnerable groups. We hope that our findings may provide a reference point for these efforts and further studies.

\section{CONCLUSION}

The current study underlines the importance of determining the more vulnerable populations for developing psychological sequelae of the COVID-19 outbreak. Female gender, younger age, being single, history of psychiatric illness, accompanying chronic diseases, lower education level and lower income were the risk factors for negative psychiatric outcomes. Moreover, perceived social support especially from the family influences the mental health of the population. 
The most significant impacts on public mental health are likely to be seen after the outbreak ends. Mental health policy considerations should focus on the unmet and anticipated needs of vulnerable populations and the mental health systems. Our study suggests that post COVID-19 mental health policies should stress the importance of personal vulnerabilities for having psychological symptoms and the positive effect of social support on these symptoms as well. Developing better ways for social media to have more helpful effects than its current detrimental effects on psychological wellbeing is also needed.

\section{Acknowledgements:}

We thank all of the participants in this study.

\section{Funding:}

This research did not receive any specific grant from funding agencies in the public, commercial, or not-forprofit sectors.

\section{Conflict of interest: None to declare.}

\section{Contribution of individual authors:}

Hatice Kaya, Batuhan Ayık \& Rumeysa Tasdelen involved in the study design, investigation and writing the initial draft.

Mete Ercis carried out the formal analysis of the data and wrote the results section of this article.

Erhan Ertekin involved in the study design, reviewed the article, and prepared the revision.

\section{References}

1. Ahmed MZ, Ahmed O, Aibao Z, Hanbin S, Siyu L \& Ahmad A: Epidemic of COVID-19 in China and Associated Psychological Problems. Asian J Psychiatr 2020; 51:102092

2. Antony MM, Cox BJ, Enns MW, Bieling PJ \& Swinson RP: Psychometric Properties of the 42-Item and 21-Item Versions of the Depression Anxiety Stress Scales in Clinical Groups and a Community Sample. Psychol Assess, 1998

3. Cao W, Fang Z, Hou G, Han $M, X u X$, Dong $J$ et al.: The Psychological Impact of the COVID-19 Epidemic on College Students in China. Psychiatry Res 2020; 287:112934

4. Chew QH, Wei KC, Vasoo S, Chua HC \& Sim K: Narrative Synthesis of Psychological and Coping Responses towards Emerging Infectious Disease Outbreaks in the General Population: Practical Considerations for the COVID-19 Pandemic. Singapore Med J 2020; 61:350-6

5. Chong MY, Wang WC, Hsieh WC, Lee CY, Chiu NM, Yeh WC et al.: Psychological Impact of Severe Acute Respiratory Syndrome on Health Workers in a Tertiary Hospital. Br J Psychiatry 2004; 185:127-33

6. De Sousa A, Mohandas E \& Javed A: Psychological Interventions during COVID-19: Challenges for Low and Middle Income Countries. Asian J Psychiatr 2020; 102128:51
7. Eker D \& Arkar H: Çok Boyutlu Algılanan Sosyal Destek Ölçeği'nin Faktör Yapısı, Geçerlik ve Güvenirliği. Türk Psikoloji Dergisi 1995;10:45-55

8. Gershon R, Dernehl LA, Nwankwo E, Zhi Q \& Qureshi K: Experiences and Psychosocial Impact of West Africa Ebola Deployment on US Health Care Volunteers. PLoS Curr 2016; 8

9. Guan $W, \mathrm{Ni} Z, \mathrm{Hu} Y$, Liang $W$, Ou C, He J et al.: Clinical Characteristics of Coronavirus Disease 2019 in China. N Engl J Med 2020; 382:1708-20

10. Hao F, Tan W, Jiang L, Zhang L, Zhao X, Zou Y et al.: Do Psychiatric Patients Experience More Psychiatric Symptoms during COVID-19 Pandemic and Lockdown? A Case-Control Study with Service and Research Implications for Immunopsychiatry. Brain Behav Immun 2020; 87:100-6

11. Holmes EA, O'Connor RC, Perry VH, Tracey I, Wessely S, Arseneault $L$ et al: Multidisciplinary research priorities for the COVID-19 pandemic: a call for action for mental health science. The Lancet Psychiatry 2020; 7:547-60

12. Hu Z, Yang Z, Li Q, Zhang A \& Huang Y: Infodemiological Study on COVID-19 Epidemic and COVID-19 Infodemic. Preprints 2020

13. Huang $Y \&$ Zhao N: Mental Health Burden for the Public Affected by the COVID-19 Outbreak in China: Who Will Be the High-Risk Group? Psychol Heal Med 2020; 14:1-12

14. Huang $Y$ \& Zhao N: Generalized Anxiety Disorder, Depressive Symptoms and Sleep Quality during COVID-19 Outbreak in China: A Web-Based Cross-Sectional Survey Psychiatry Res 2020; 288:112954

15. Jakovljevic $M$, Bjedov $S$, Jaksic $N$ \& Jakovljevic I: COVID-19 Pandemia and Public and Global Mental Health from the Perspective of Global Health Security. Psychiatr Danub 2020; 32:6-14

16. Kawohl $W$ \& Nordt C: COVID-19, Unemployment, and Suicide. The Lancet Psychiatry 2020; 5:389-390

17. Ko NY, Lu WH, Chen YL, Li DJ, Wang PW, Hsu ST et al.: COVID-19-Related Information Sources and Psychological Well-Being: An Online Survey Study in Taiwan. Brain, Behavior, and Immunity 2020; 87:153-4

18. Lei L, Huang X, Zhang S, Yang J, Yang L \& Xu M: Comparison of Prevalence and Associated Factors of Anxiety and Depression among People Affected by versus People Unaffected by Quarantine during the COVID-19 Epidemic in Southwestern China Med Sci Monit 2020; 26:e924609-1

19. Liu N, Zhang F, Wei C, Jia Y, Shang Z, Sun L et al.: Prevalence and Predictors of PTSS during COVID-19 Outbreak in China Hardest-Hit Areas: Gender Differences Matter. Psychiatry Res 2020; 287:112921

20. Lovibond SH \& Lovibond PF: Manual for the Depression Anxiety Stress Scales, 1995

21. Özdin $S \&$ Bayrak Özdin Ş: Levels and Predictors of Anxiety, Depression and Health Anxiety during COVID-19 Pandemic in Turkish Society: The Importance of Gender. Int J Soc Psychiatry 2020; 66:504-11

22. Peteet JR: COVID-19 Anxiety. J Relig Health 2020; 59:2203-4

23. Rajkumar RP: COVID-19 and Mental Health: A Review of the Existing Literature. Asian J Psychiatr 2020; 52:102066

24. Republic of Turkey Ministry of Health: Timeline of COVID-19 outbreak https://covid19.saglik.gov.tr/ (accessed Jul 1, 2020) 
25. Reznik A, Gritsenko V, Konstantinov V, Khamenka $N \&$ Isralowitz R: COVID-19 Fear in Eastern Europe: Validation of the Fear of COVID-19 Scale. International Journal of Mental Health and Addiction 2020; 12:1-6

26. Torales J, O'Higgins M, Castaldelli-Maia JM \& Ventriglio A: The Outbreak of COVID-19 Coronavirus and Its Impact on Global Mental Health. International Journal of Social Psychiatry 2020; 66:317-20

27. Wang C, Pan R, Wan X, Tan Y, Xu L, Ho CS et al.: Immediate Psychological Responses and Associated Factors during the Initial Stage of the 2019 Coronavirus Disease (COVID-19) Epidemic among the General Population in China. Int J Environ Res Public Health 2020; 17:1729

28. WHO (World Health Organization): Statement on the Meeting of the International Health Regulations (2005) Emergency Committee Regarding the Outbreak of Novel Coronavirus (2019-NCoV). WHO Newsletter 2020; 1-6

29. Xiang YT, Jin Y \& Cheung T: Joint International Collaboration to Combat Mental Health Challenges during the Coronavirus Disease 2019 Pandemic. JAMA Psychiatry. 2020. Online ahead of print

30. Xiao H, Zhang Y, Kong D, Li S \& Yang N: The Effects of Social Support on Sleep Quality of Medical Staff Treating Patients with Coronavirus Disease 2019 (COVID-19) in January and February 2020 in China. Med Sci Monit 2020; 26:e923549-1

31. Yelin D, Wirtheim E, Vetter P, Kalil AC, Bruchfeld J, Runold $M$ et al.: Long-term consequences of COVID-19: research needs. The Lancet Infectious Diseases 2020; 20:1115-7

32. Yeung DYL \& Fung HH: Age Differences in Coping and Emotional Responses toward SARS: A Longitudinal Study of Hong Kong Chinese. Aging Ment Heal 2007; 11:579587

33. Yllmaz Ö, Boz $H \&$ Arslan A: The Validity and Reliability of Depression Stress and Anxiety Scale (Dass-21) Turkish Short Form. J Financ Econ Soc Res 2017; 78-91

34. Yoo JH: The Fight against the 2019-NCoV Outbreak: An Arduous March Has Just Begun. Journal of Korean Medical Science 2020; 35:56

35. Zhang J, Lu H, Zeng H, Zhang S, Du Q, Jiang Tet al.: The Differential Psychological Distress of Populations Affected by the COVID-19 Pandemic. Brain, Behavior, and Immunity 2020; 87:49-50

36. Zhou F, Yu T, Du R, Fan G, Liu Y, Liu Z et al.: Clinical Course and Risk Factors for Mortality of Adult Inpatients with COVID-19 in Wuhan, China: A Retrospective Cohort Study. The Lancet Psychiatry 2020; 395:1054-62

37. Zhu S, Wu Y, Zhu C, Van Hong W. Chu Yu Z, Xi Chen et al.: The Immediate Mental Health Impacts of the COVID19 Pandemic among People with or without Quarantine Managements. Brain, Behavior, and Immunity 2020; 87:56-8

38. Zimet GD, Dahlem NW, Zimet $S G$ \& Farley GK: The Multidimensional Scale of Perceived Social Support. J Pers Assess 1988; 52:30-41
Correspondence:

Hatice Kaya, MD

Department of Psychiatry, Istanbul Sultanbeyli State Hospital, Sultanbeyli Community Mental Health Center

Istanbul, Sultanbeyli, Turkey

E-mail: doc.haticekaya@gmail.com 